\title{
Linear-quadratic optimal boundary control of a one-link flexible arm
}

\author{
Andrea Cristofaro ${ }^{\star}$, Alessandro De Luca, Leonardo Lanari
}

\begin{abstract}
A linear-quadratic optimal control problem is considered for the infinite-dimensional model of a one-link flexible arm. Two boundary inputs are assumed to be available, namely the joint torque at the link base and a transverse force at the tip of the link. The problem is formulated and solved using semigroup theory and duality arguments. Simulation results are provided to support the theoretical findings, comparing the proposed optimal LQ law with a more conventional PD/state feedback controller in terms of cost and transient performance.
\end{abstract}

Index Terms-Distributed parameter systems; Robotics; Optimal control

\section{INTRODUCTION}

C ONTROLLED mechanical systems with flexible components have recently become an important and fertile research area, due to their versatility, high speed response and low energy consumption. Several examples of such systems may be found in soft robotics, i.e., flexible manipulators [1] or UAVs with flexible and articulated wings [2]. These systems are characterized by a distributed parameter nature, and their dynamics are typically governed by a combination of highorder partial differential equations (PDEs), ordinary differential equations (ODEs) and a set of static boundary conditions. Coupled systems of first and second order PDEs with ODEs have been largely investigated in the literature, and tackled with different approaches, such as backstepping [3], [4] or Lyapunov methods [5], [6], [7].

A complete description of finite-dimensional models of a single-link flexible robotic arm with geometric and dynamic boundary conditions is provided in [8], while additional insights on the relation between finite- and infinite-dimensional models of a flexible beam are given in [9]. The system has been recognized to have non-minimum phase zeros that depend on the inertia at the link end, and some techniques to overcome this issue have been proposed [10], [11]. Simultaneous stabilization of beam orientation and deflection was investigated in [12], [13], while the trajectory tracking problem was addressed, e.g., in [14], [15], [16], [17].

In this paper, we consider a flexible beam carrying a mass with inertia at the tip and clamped at the base to a motor providing a joint torque. An additional actuator is placed at the tip, generating an input force in the transverse direction to the beam axis (see, e.g., [10] for an actual implementation of such command). In particular, such input redundancy can be used to achieve secondary objectives e.g., reduction of oscillations and/or improvement of tracking accuracy.

The classical Euler-Bernoulli infinite-dimensional model is

${ }^{\star}$ Corresponding author, andrea.cristofaro@diag.uniromal.it Authors' names are listed in alphabetical order. The authors are with the Department of Computer, Control and Management Engineering, Sapienza University of Rome, Via Ariosto 25, 00185 Rome, Italy. adopted for the flexible beam. Despite some potential increase in computational complexity, dynamical models based on PDEs guarantee higher accuracy in reflecting the distributed characteristics of the system, as compared to finitedimensional approximations based on the assumed modes description. Moreover, using operator theory, the considered model can be successfully translated into an abstract linear differential system defined on a suitable Hilbert space and, as such, it can be treated using some tools inherited from classical and optimal control theory [18], [19].

We focus in particular on a class of linear-quadratic regulation problems for the Euler-Bernoulli model of a singlelink flexible arm, and we look for the optimal inputs capable of transferring the system between two equilibrium states, both with zero link deformation, from an initial to a final desired joint position (rest-to-rest slewing maneuver). To the best of our knowledge, this problem has never been explicitly addressed before in the infinite-dimensional framework.

The main contributions of the paper can be summarized as follows.

- We introduce a quadratic cost functional that weights the joint angle position error, the distributed deflection of the beam during motion, and in particular the link deformation at the tip, as well as the control efforts.

- The optimal control commands are obtained as linear functions of the system co-state, which satisfies an adjoint equation defined in a natural way.

- The results obtained with the proposed optimal LQ framework are compared with a controller that uses a standard PD law for the joint torque and a state feedback stabilizing law for the tip force.

Some related results, with a specific emphasis on the finitedimensional approximation of linear-quadratic optimal controls via modal decomposition, can be found in [20], where the authors claim an interesting property of commutativity for the operations of discretization and optimization.

The paper is organized as follows. The basic notation and the problem setup are given in Section II, while the wellposedness is addressed in Section III. Having guaranteed the existence of solutions, the actual optimal control problem is tackled in Section IV. Finally, a numerical case study is considered in Section V.

\section{Problem Setup}

Let us consider a single-link flexible robotic arm of length $L>0$, with the link base attached to an actuated joint and the free end carrying a payload. Without loss of generality, the motion is assumed to be constrained on a horizontal plane, thus neutralizing the gravity action. We denote by $\varrho$ the mass density and by $E I$ the flexural rigidity of the link, by $M_{p}$ the 


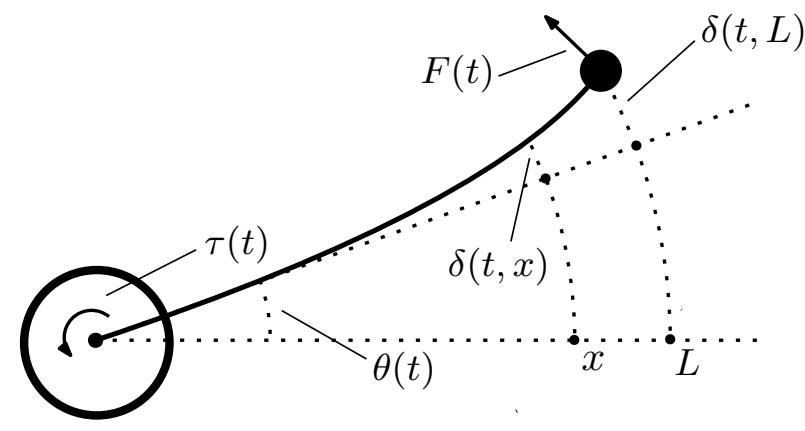

Figure 1. Sketch of the one-link flexible arm.

tip mass, and by $J_{h}$ and $J_{p}$ the inertia of the base motor and of the tip payload, respectively. Figure 1 shows a sketch of the flexible arm with its control inputs $\tau$ and $F$. The dynamics of the flexible arm is described by the following linear partial differential equation based on the well-known Euler-Bernoulli beam model [1], [13]

$$
\varrho \ddot{z}(t, x)=-E I z_{x x x x}(t, x) \quad x \in(0, L)
$$

with boundary conditions

$$
\begin{aligned}
z(t, 0) & =0 \\
J_{h} \ddot{z}_{x}(t, 0)-E I z_{x x}(t, 0) & =\tau(t) \\
M_{p} \ddot{z}(t, L)-E I z_{x x x}(t, L) & =F(t) \\
J_{p} \ddot{z}_{x}(t, L)+E I z_{x x}(t, L) & =0
\end{aligned}
$$

In the equations above, the dot notation $\dot{z}$ stands for partial derivative with respect to $t$ (time), while the subscript $z_{x}$ indicates partial derivative with respect to $x$ (space). The state of the system is $z(t, x)=x \theta(t)+\delta(t, x)$, where $\theta(t)$ is the joint angle and $\delta(t, x)$ represents the distributed beam deflection. The tip force $F(t)$ and the joint torque $\tau(t)$ are the accessible control inputs to the system. The torque at the base $\tau(t)$ is a typical control input in robotic systems, whereas the tip force, following [1], [10], is somewhat redundant but allows to better balance the arm deflection induced by the joint rotation and reduce oscillations. Fixing a finite time horizon $[0, T]$ and a reference trajectory profile for the joint angle $\theta_{d}(t)$, we are typically interested in determining optimal control inputs $F^{\star}(t), \tau^{\star}(t)$ that attain minimization of quadratic cost indices of the form

$$
\begin{gathered}
\left(F^{\star}(t), \tau^{\star}(t)\right)=\arg \min W(F, \tau) \\
W(F, \tau)=\int_{0}^{T}\left(\alpha \int_{0}^{L}|\delta(t, x)|^{2} d x+\beta\left|\theta(t)-\theta_{d}(t)\right|^{2}\right) d t \\
+\alpha_{L} \int_{0}^{T}|\delta(t, L)|^{2} d t+\int_{0}^{T}[F(t) \tau(t)] \Gamma\left[\begin{array}{c}
F(t) \\
\tau(t)
\end{array}\right] d t \\
+\alpha_{T} \int_{0}^{L}|\delta(T, x)|^{2} d x+\beta_{T}\left|\theta(T)-\theta_{d}(T)\right|^{2}
\end{gathered}
$$

with weights $\alpha, \beta, \alpha_{L}, \alpha_{T}, \beta_{T} \geq 0$ and $\boldsymbol{\Gamma}=\boldsymbol{\Gamma}^{T} \succ 0$. The minimization of the cost (3) aims at reducing the deflection along the whole arm while tracking the desired joint angle trajectory and simultaneously attempting to keep the control effort low. In particular, independent weights are assigned to the total deflection $(\alpha)$, the joint tracking error $(\beta)$, the deflection at the tip $\left(\alpha_{L}\right)$, and the configuration at the final time $T\left(\alpha_{T}, \beta_{T}\right)$.

The PDE problem (1-2) can be rearranged in an abstract form using operator theory. In particular, reordering and merging the boundary states in a single vector, the system can be written in the equivalent form

$$
\dot{\xi}=\mathcal{A} \boldsymbol{\xi}+\mathcal{B} \boldsymbol{v}
$$

where $\boldsymbol{\xi}=\left[\begin{array}{ll}\boldsymbol{\xi}_{1}^{T} & \boldsymbol{\xi}_{2}^{T}\end{array}\right]^{T}$ is the state with

$$
\begin{aligned}
& \boldsymbol{\xi}_{1}=\left[z(t, x) z_{x}(t, L) z(t, L) z_{x}(t, 0)\right]^{T} \\
& \boldsymbol{\xi}_{2}=\dot{\boldsymbol{\xi}}_{1}
\end{aligned}
$$

and $\boldsymbol{v}=[F(t) \tau(t)]^{T}$ is the boundary input vector. It is worth to highlight that the first and and the fifth components of the state vector $\boldsymbol{\xi}$, i.e., $z(t, x)$ and $\dot{z}(t, x)$, are distributed quantities and therefore infinite-dimensional states. The operators $\mathcal{A}, \mathcal{B}$ are given, respectively, by

$$
\mathcal{A}=\left[\begin{array}{cc}
\mathbf{0}_{4 \times 4} & \boldsymbol{I}_{4 \times 4} \\
-\mathcal{A}_{0} & \mathbf{0}_{4 \times 4}
\end{array}\right], \quad \mathcal{B}=\left[\begin{array}{cc}
\mathbf{0}_{6 \times 1} & \mathbf{0}_{6 \times 1} \\
M_{p}^{-1} & 0 \\
0 & J_{h}^{-1}
\end{array}\right]
$$

with

$$
-\mathcal{A}_{0} \boldsymbol{\xi}_{1}=\left[\begin{array}{r}
-\sigma z_{x x x x}(t, x) \\
-\epsilon_{1} z_{x x}(t, L) \\
\epsilon_{2} z_{x x x}(t, L) \\
\epsilon_{3} z_{x x}(t, 0)
\end{array}\right]
$$

and where coefficients have been rearranged as $\sigma=\varrho^{-1} E I$, $\epsilon_{1}=J_{p}^{-1} E I, \epsilon_{2}=M_{p}^{-1} E I$, and $\epsilon_{3}=J_{h}^{-1} E I$.

Let us recall that $L^{2}(0, L)$ indicates the space of functions $f(x)$ such that the integral $\int_{0}^{L}|f(x)|^{2} d x$ exists and is finite. Moreover, the Sobolev space $H^{k}(0, L)$ is the space of functions $f(x)$ whose derivatives of order $j=0,1, \ldots, k$ are in $L^{2}(0, L)$. Introducing then the subset

$$
H_{0^{-}}^{k}(0, L):=\left\{z \in H^{k}(0, L): z(0)=0\right\}
$$

to take into account the boundary condition at the base, we can define the state space for system (4) as the Hilbert space

$$
\mathcal{H}:=H_{0^{-}}^{2}(0, L) \times \mathbb{R}^{3} \times \mathcal{V}
$$

with $\mathcal{V}=L^{2}(0, L) \times \mathbb{R}^{3}$, endowed with the inner product

$$
\begin{aligned}
\langle\boldsymbol{\xi}, \tilde{\boldsymbol{\xi}}\rangle_{\mathcal{H}}= & \left\langle\mathcal{A}_{0} \boldsymbol{\xi}_{1}, \tilde{\boldsymbol{\xi}}_{1}\right\rangle_{\mathcal{V}}+\left\langle\boldsymbol{\xi}_{2}, \tilde{\boldsymbol{\xi}}_{2}\right\rangle_{\mathcal{V}} \\
& +z_{x}(0) \tilde{z}_{x}(0)+\zeta_{3} \tilde{\zeta}_{3}
\end{aligned}
$$

for $\boldsymbol{\xi}=\left(\boldsymbol{\xi}_{1}, \boldsymbol{\xi}_{2}\right)=(z, \boldsymbol{\zeta}, w, \boldsymbol{\omega}), \quad \tilde{\boldsymbol{\xi}}=\left(\begin{array}{ll}\tilde{\boldsymbol{\xi}}_{1}, & \tilde{\boldsymbol{\xi}}_{2}\end{array}\right)_{\tilde{\tau}}=$ $(\tilde{z}, \tilde{\boldsymbol{\zeta}}, \tilde{w}, \tilde{\boldsymbol{\omega}}) \in \mathcal{H}$, where $\boldsymbol{\zeta}=\left(\zeta_{1}, \zeta_{2}, \zeta_{3}\right)$ and $\tilde{\boldsymbol{\zeta}}=\left(\tilde{\zeta}_{1}, \tilde{\zeta}_{2}, \tilde{\zeta}_{3}\right)$. Accordingly, the domain of the operator $\mathcal{A}$ is the subspace

$$
\begin{array}{r}
\mathcal{D}(\mathcal{A})=\left\{(z, \boldsymbol{\zeta}, w, \boldsymbol{\omega}) \in H_{0^{-}}^{4}(0, L) \times \mathbb{R}^{3} \times H_{0^{-}}^{2}(0, L) \times \mathbb{R}^{3}:\right. \\
\zeta_{1}=z_{x}(L), \zeta_{2}=w(L), \zeta_{3}=z_{x}(0), \\
\left.\omega_{1}=w_{x}(L), \omega_{2}=w(L), \omega_{3}=w_{x}(0)\right\}
\end{array}
$$

It is easy to see that $\mathcal{D}(\mathcal{A})$ is dense in $\mathcal{H}$. The inner product defined in (10) induces a norm on the Hilbert space $\mathcal{H}$ that corresponds to a classical energy norm (potential energy + kinetic energy) plus some correction terms to evaluate the rigid motion $z_{x}(0)$ (see [13] for further details). 


\section{Well POSEDNESS}

In order to design an optimal controller for the flexible arm, we need to guarantee that system (4) is well posed, this meaning that for any admissible control input $\boldsymbol{v}(t)$ there exists a unique solution $\boldsymbol{\xi}(t)$. In this regard, let us state the main theorem of this section.

Theorem 3.1: The operator $\mathcal{A}: \mathcal{D}(\mathcal{A}) \rightarrow \mathcal{H}$ generates a strongly continuous semigroup $\mathcal{T}(t)=e^{\mathcal{A} t}$ on the space $\mathcal{H}$.

The forthcoming derivations are instrumental for the proof of the theorem. For $\boldsymbol{\xi} \in \mathcal{D}(\mathcal{A})$, let us evaluate

$$
\begin{aligned}
\langle\mathcal{A} \boldsymbol{\xi}, \boldsymbol{\xi}\rangle_{\mathcal{H}} & =\left\langle\mathcal{A}_{0} \boldsymbol{\xi}_{2}, \boldsymbol{\xi}_{1}\right\rangle_{\mathcal{V}}+\left\langle-\mathcal{A}_{0} \boldsymbol{\xi}_{1}, \boldsymbol{\xi}_{2}\right\rangle_{\mathcal{V}} \\
& +w_{x}(0) z_{x}(0)+\omega_{3} \zeta_{3}
\end{aligned}
$$

Observing that $\boldsymbol{A}_{0}$ is self-adjoint, i.e. $\left\langle-\mathcal{A}_{0} \boldsymbol{\xi}_{1}, \boldsymbol{\xi}_{2}\right\rangle_{\mathcal{V}}=$ $-\left\langle\boldsymbol{\xi}_{1}, \mathcal{A}_{0} \boldsymbol{\xi}_{2}\right\rangle_{\mathcal{V}}$, and using $\boldsymbol{\xi} \in \mathcal{D}(\mathcal{A})$ one gets

$$
\langle\mathcal{A} \boldsymbol{\xi}, \boldsymbol{\xi}\rangle_{\mathcal{H}}=2 \omega_{3} \zeta_{3} \leq\langle\boldsymbol{\xi}, \boldsymbol{\xi}\rangle_{\mathcal{H}}
$$

where Schwartz inequality has been applied. Consider now the adjoint operator $\mathcal{A}^{*}$, together with its domain $\mathcal{D}\left(\mathcal{A}^{*}\right)$, such that

$$
\langle\mathcal{A} \boldsymbol{\xi}, \boldsymbol{\nu}\rangle_{\mathcal{H}}=\left\langle\boldsymbol{\xi}, \mathcal{A}^{*} \boldsymbol{\nu}\right\rangle_{\mathcal{H}} \quad \forall \boldsymbol{\xi} \in \mathcal{D}(\mathcal{A}), \forall \boldsymbol{\nu} \in \mathcal{D}\left(\mathcal{A}^{*}\right)
$$

Using standard duality arguments and the self-adjointness of the operator $\mathcal{A}_{0}$, it follows that, given $\boldsymbol{\nu}=(y, \boldsymbol{\psi}, u, \boldsymbol{\eta}) \in$ $\mathcal{D}\left(\mathcal{A}^{*}\right) \subset \mathcal{H}$, the adjoint of $\mathcal{A}$ has the expression

$$
\mathcal{A}^{*}=\left[\begin{array}{cc}
\mathbf{0}_{4 \times 4} & -\boldsymbol{I}_{4 \times 4} \\
\mathcal{A}_{0} & \mathbf{0}_{4 \times 4}
\end{array}\right]
$$

with domain

$$
\begin{array}{r}
\mathcal{D}\left(\mathcal{A}^{*}\right)=\left\{(y, \boldsymbol{\psi}, u, \boldsymbol{\eta}) \in H_{0^{-}}^{4}(0, L) \times \mathbb{R}^{3} \times H_{0^{-}}^{2}(0, L) \times \mathbb{R}^{3}:\right. \\
\psi_{1}=-y_{x}(L), \psi_{2}=-y(L), \psi_{3}=-y_{x}(0), \\
\left.\eta_{1}=-u_{x}(L), \eta_{2}=-u(L), \eta_{3}=-u_{x}(0)\right\}
\end{array}
$$

In particular for $\boldsymbol{\nu}=\left(\boldsymbol{\nu}_{1}, \boldsymbol{\nu}_{2}\right)$, by direct calculation, we have

$$
\begin{aligned}
\langle\mathcal{A} \boldsymbol{\xi}, \boldsymbol{\nu}\rangle_{\mathcal{H}}= & \left\langle\mathcal{A}_{0} \boldsymbol{\xi}_{2}, \boldsymbol{\nu}_{1}\right\rangle_{\mathcal{V}}+\left\langle-\mathcal{A}_{0} \boldsymbol{\xi}_{1}, \boldsymbol{\nu}_{2}\right\rangle_{\mathcal{V}} \\
& +\underbrace{w_{x}(0) y_{x}(0)+\omega_{3} \psi_{3}}_{=0} \\
= & \left\langle\mathcal{A}_{0}^{\left.\boldsymbol{A}_{1},-\boldsymbol{\nu}_{2}\right\rangle_{\mathcal{V}}+\left\langle\boldsymbol{\xi}_{2}, \mathcal{A}_{0} \boldsymbol{\nu}_{1}\right\rangle_{\mathcal{V}}}\right. \\
\underbrace{-z_{x}(0) u_{x}(0)-\zeta_{3} \eta_{3}}_{=0} & =\left\langle\boldsymbol{\xi}, \mathcal{A}^{*} \boldsymbol{\nu}\right\rangle_{\mathcal{H}}
\end{aligned}
$$

We are now ready to prove the well-posedness of the differential system (4).

Proof: [of Theorem 3.1] Evaluating the product $\left\langle\mathcal{A}^{*} \boldsymbol{\nu}, \boldsymbol{\nu}\right\rangle_{\mathcal{H}}$, and applying again Schwartz inequality, yields

$$
\left\langle\mathcal{A}^{*} \boldsymbol{\nu}, \boldsymbol{\nu}\right\rangle_{\mathcal{H}}=-u_{x}(0) y_{x}(0)-\eta_{3} \psi_{3} \leq\langle\boldsymbol{\nu}, \boldsymbol{\nu}\rangle_{\mathcal{H}}
$$

The conclusion then follows by applying [18, Corollary 2.2.3]. In particular, the semigroup $\mathcal{T}(t)$ generated by the operator $\mathcal{A}$ satisfies

$$
\|\boldsymbol{T}(t)\| \leq e^{c_{0} t}
$$

The unique solution of (4) is in the form $\boldsymbol{\xi}(t)=e^{\mathcal{A} t} \boldsymbol{\xi}(0)+$ $e^{\mathcal{A} t} * \mathcal{B} \boldsymbol{v}(t)$, where $*$ stands for the convolution product.

\section{Optimal Control}

In order to guarantee existence and uniqueness of the optimal control, it is desirable to have the running cost and the terminal cost in the form of the squared $\mathcal{H}$-norm induced by (10) (see for instance [21]). This is not the case for the functional $W(F, \tau)$ defined in (3): although this seems natural and intuitive choice for the cost index, it is not suitable for the infinite-dimensional LQR theory. To overcome the issue, we can consider the relaxed class of problems

$$
\begin{gathered}
\left(F^{\star}(t), \tau^{\star}(t)\right)=\arg \min \bar{W}(F, \tau) \\
\bar{W}(F, \tau)=\int_{0}^{T}\left\langle\left(\boldsymbol{\xi}(t)-\boldsymbol{\xi}_{d}(t)\right), \boldsymbol{\Lambda}\left(\boldsymbol{\xi}(t)-\boldsymbol{\xi}_{d}(t)\right)\right\rangle_{\mathcal{H}} d t \\
+\int_{0}^{T}[F(t) \tau(t)] \boldsymbol{\Gamma}\left[\begin{array}{c}
F(t) \\
\tau(t)
\end{array}\right] d t \\
+\left\langle\left(\boldsymbol{\xi}(T)-\boldsymbol{\xi}_{d}(T)\right), \boldsymbol{K}\left(\boldsymbol{\xi}(T)-\boldsymbol{\xi}_{d}(T)\right)\right\rangle_{\mathcal{H}}
\end{gathered}
$$

where $\boldsymbol{\Lambda}=\boldsymbol{\Lambda}^{T} \succeq 0, \boldsymbol{K}=\boldsymbol{K}^{T} \succeq 0$ are constant matrices in $\mathbb{R}^{8 \times 8}$ and $\boldsymbol{\xi}_{d}(t) \in L^{2}((0, T) ; \mathcal{H})$ is a state reference trajectory. The first integral term in (18) weights the state tracking error, the second integral term weights the control effort (this is left unchanged from (3)) and the last term represents a terminal cost. The weighting functions of states and inputs appearing in the integral terms are referred to as the running cost.

Remark 4.1: The relaxed problem (18) can be brought to a form similar to the original one (3), but including a mixed term depending on $\theta(t)$ and $\delta(t, x)$. This can be done selecting the weighting matrices as

$$
\begin{aligned}
& \boldsymbol{\Lambda}=\operatorname{diag}\left\{\alpha, \beta / 2, \alpha_{L}, \beta / 2,0,0,0,0\right\} \succeq 0 \\
& \boldsymbol{K}=\operatorname{diag}\left\{\alpha_{T}, \beta_{T} / 2,0, \beta_{T} / 2,0,0,0,0\right\} \succeq 0
\end{aligned}
$$

and considering a reference trajectory

$\boldsymbol{\xi}_{d}(t)=\left(z_{d}(t, x), z_{x, d}(t, L), z_{d}(t, L), z_{x, d}(t, 0), \ldots, \dot{z}_{x, d}(t, 0)\right)$ with

$$
\begin{array}{ll}
z_{d}(t, x)=x \theta_{d}(t) & z_{x, d}(t, L)=\theta_{d}(t) \\
z_{d}(t, L)=L \theta_{d}(t) & z_{x, d}(t, 0)=\theta_{d}(t)
\end{array}
$$

which corresponds to a prescribed trajectory for the joint angle $\theta_{d}(t)$ and to an identically zero deflection $\delta_{d}(t, x) \equiv 0$.

\section{A. The adjoint equation}

The infinite-dimensional system (4) is described by an unbounded state operator $\mathcal{A}$ together with a bounded input operator $\mathcal{B}$, and therefore the LQ optimal control synthesis technique described in [21, Chapter 7] can be applied. To this end, we introduce the adjoint equation to (4)

$$
-\dot{\boldsymbol{p}}=\mathcal{A}^{*} \boldsymbol{p}+\boldsymbol{g}, \quad \boldsymbol{p}(T)=\boldsymbol{p}_{T}
$$

where $\left(\boldsymbol{g}, \boldsymbol{p}_{T}\right) \in L^{2}((0, T) ; \mathcal{H}) \times \mathcal{H}$ are arbitrary. As long as $\xi$ is a solution to

$$
\dot{\xi}=\mathcal{A} \boldsymbol{\xi}+\boldsymbol{f}, \quad \boldsymbol{\xi}(0)=\boldsymbol{\xi}_{0}
$$

a useful duality argument is in force. 
Lemma 4.1: For every $\left(\boldsymbol{f}, \boldsymbol{\xi}_{0}\right) \in L^{2}((0, T) ; \mathcal{H}) \times \mathcal{H}$ and every $\left(\boldsymbol{g}, \boldsymbol{p}_{T}\right) \in L^{2}((0, T) ; \mathcal{H}) \times \mathcal{H}$ the following formula holds true

$$
\begin{aligned}
\int_{0}^{T}\langle\boldsymbol{f}(t), \boldsymbol{p}(t)\rangle_{\mathcal{H}} d t & =\int_{0}^{T}\langle\boldsymbol{g}(t), \boldsymbol{\xi}(t)\rangle_{\mathcal{H}} d t \\
& +\left\langle\boldsymbol{\xi}(T), \boldsymbol{p}_{T}\right\rangle_{\mathcal{H}}-\left\langle\boldsymbol{\xi}_{0}, \boldsymbol{p}(0)\right\rangle_{\mathcal{H}}
\end{aligned}
$$

The proof uses properties of differentiation under the integral sign and density arguments [21]. While the above duality formula holds in general, the design of the optimal controller in closed-form requires a special choice for the function $\boldsymbol{g}$ and the final condition $\boldsymbol{p}_{T}$. Similar to the finite-dimensional case, $\boldsymbol{g}$ and $\boldsymbol{p}_{T}$ have to be selected as the derivatives ${ }^{1}$ of the running cost and the terminal cost, respectively. Such a choice leads to the adjoint infinite-dimensional system

$$
\begin{gathered}
-\dot{\boldsymbol{p}}=\mathcal{A}^{*} \boldsymbol{p}-2 \boldsymbol{\Lambda}\left(\boldsymbol{\xi}-\boldsymbol{\xi}_{d}\right) \\
\boldsymbol{p}(T)=-2 \boldsymbol{K}\left(\boldsymbol{\xi}(T)-\boldsymbol{\xi}_{d}(T)\right)
\end{gathered}
$$

\section{B. Optimal control synthesis}

Let us preliminary observe that the existence of the optimal control follows from the weak compactness of minimizing sequences and from the weak lower semi-continuity of the cost functional [21, Theorem 7.3.1], which is guaranteed by the convexity of the running cost and the terminal cost. Furthermore, convexity can also be used to prove uniqueness of the optimal control. The following statement addresses the problem of optimal control synthesis.

Theorem 4.1: Consider equation (4) coupled with the adjoint equation (24). Let $\left(\boldsymbol{\xi}_{\mathrm{opt}}, \boldsymbol{v}_{\mathrm{opt}}\right)$ be the optimal solution for system (4) with the cost functional (18). Then the optimal input $\boldsymbol{v}_{\mathrm{opt}}$ is given by

$$
\boldsymbol{v}_{\mathrm{opt}}=-\boldsymbol{\Gamma}^{-1} \mathcal{B}^{T} \boldsymbol{p}_{\mathrm{opt}}
$$

where $\boldsymbol{p}_{\mathrm{opt}}$ is the solution to the adjoint equation (24) with the optimal state $\boldsymbol{\xi}_{\mathrm{opt}}$.

Proof: Let $\left(\boldsymbol{\xi}_{\text {opt }}, \boldsymbol{v}_{\text {opt }}\right)$ be the optimal solution, and set $G(\boldsymbol{v})=\bar{W}^{\prime}(\boldsymbol{v})$, where the derivative is performed in the Fréchet sense. By optimality, we must have $G\left(\boldsymbol{v}_{\text {opt }}\right)=0$. On the other hand, for any $\boldsymbol{v} \in L^{2}(0, T) \times L^{2}(0, T)$, the following holds

$$
\begin{aligned}
G\left(\boldsymbol{v}_{\mathrm{opt}}\right) \boldsymbol{v}= & \int_{0}^{T}\left\langle 2 \Lambda\left(\boldsymbol{\xi}_{\mathrm{opt}}-\boldsymbol{\xi}_{d}\right), \boldsymbol{\chi}\right\rangle_{\mathcal{H}} d t+\int_{0}^{T} \boldsymbol{v}_{\mathrm{opt}}^{T} \boldsymbol{\Gamma} \boldsymbol{v} d t \\
& +\left\langle 2 \boldsymbol{K}\left(\boldsymbol{\xi}_{\mathrm{opt}}(T)-\boldsymbol{\xi}_{d}(T)\right), \boldsymbol{\chi}(T)\right\rangle_{\mathcal{H}}
\end{aligned}
$$

where $\chi$ is the solution to

$$
\begin{aligned}
\dot{\chi} & =\mathcal{A} \chi+\mathcal{B} \boldsymbol{v} \\
\chi(0) & =\mathbf{0}
\end{aligned}
$$

\footnotetext{
${ }^{1}$ To be performed in the Fréchet sense with respect to the system state.
}

Now, applying Lemma 4.1 to $\boldsymbol{p}_{\text {opt }}$ and $\chi$ and performing some simple algebraic manipulations, one gets

$$
\begin{aligned}
G\left(\boldsymbol{v}_{\mathrm{opt}}\right) \boldsymbol{v} & =\int_{0}^{T}\left\langle\boldsymbol{p}_{\mathrm{opt}}, \mathcal{B} \boldsymbol{v}\right\rangle_{\mathcal{H}} d t+\int_{0}^{T} \boldsymbol{v}_{\mathrm{opt}}^{T} \Gamma \boldsymbol{v} d t \\
& =\int_{0}^{T}\left(\Gamma^{-1} \mathcal{B}^{T} \boldsymbol{p}_{\mathrm{opt}}+\boldsymbol{v}_{\mathrm{opt}}\right)^{T} \Gamma \boldsymbol{v} d t=0
\end{aligned}
$$

Since the last identity must hold for every $\boldsymbol{v} \in L^{2}(0, T)$, one has necessarily $\boldsymbol{v}_{\text {opt }}$ given by (25), concluding the proof.

Remark 4.2: The same result may be formally obtained by considering the Hamiltonian

$\mathbb{H}(\boldsymbol{\xi}, \boldsymbol{v}, \boldsymbol{p})=\langle\boldsymbol{p}, \mathcal{A} \boldsymbol{\xi}+\boldsymbol{B} \boldsymbol{v}\rangle_{\mathcal{H}}-\left\langle\left(\boldsymbol{\xi}-\boldsymbol{\xi}_{d}\right), \boldsymbol{\Lambda}\left(\boldsymbol{\xi}-\boldsymbol{\xi}_{d}\right)\right\rangle_{\mathcal{H}}-\boldsymbol{v}^{T} \boldsymbol{\Gamma} \boldsymbol{v}$ and applying an infinite-dimensional version of the Pontryagin maximum principle. The optimal control $\boldsymbol{v}_{\mathrm{opt}}$ maximizes the Hamiltonian, and the optimal state and costate $\left(\boldsymbol{\xi}_{\mathrm{opt}}, \boldsymbol{p}_{\mathrm{opt}}\right)$ are related by the canonical equations

$$
\left\{\begin{array}{l}
\dot{\boldsymbol{\xi}}_{\mathrm{opt}}=\frac{\partial \mathbb{H}(\boldsymbol{\xi}, \boldsymbol{v}, \boldsymbol{p})}{\partial \boldsymbol{p}} \\
\dot{\boldsymbol{p}}_{\mathrm{opt}}=-\frac{\partial \mathbb{H}(\boldsymbol{\xi}, \boldsymbol{v}, \boldsymbol{p})}{\partial \boldsymbol{\xi}}
\end{array}\right.
$$

where the differentiation in the right-hand side is performed in the sense of the Fréchet derivative.

\section{Control implementation}

The cost function and the corresponding optimal control problems are given in a general form that allows to fully catch the behaviour of the system, which is characterized by an interplay of distributed and boundary states. In the typical collocated setup the boundary states and their temporal derivatives are the only quantities in the system to be directly measurable, by a joint encoder placed on the motor and, for instance, by a camera overlooking the plane of motion of the flexible arm. In this regard, let $\mathcal{C} \in \mathbb{R}^{6 \times 8}$ be defined as the output matrix

$$
\mathcal{C}=\left[\begin{array}{ll|ll}
\mathbf{0}_{3 \times 1} & \boldsymbol{I}_{3 \times 3} & \mathbf{0}_{3 \times 1} & \mathbf{0}_{3 \times 3} \\
\mathbf{0}_{3 \times 1} & \mathbf{0}_{3 \times 3} & \mathbf{0}_{3 \times 1} & \boldsymbol{I}_{3 \times 3}
\end{array}\right]
$$

corresponding to the removal of the distributed components of the state $\boldsymbol{\xi}(t)$, i.e., $z(t, x)$ and $\dot{z}(t, x)$, from the set of available measurements. If matrices $\Lambda$ and $\boldsymbol{K}$ in (18) are chosen as $\boldsymbol{\Lambda}=\mathcal{C}^{T} \hat{\boldsymbol{\Lambda}} \mathcal{C}$ and $\boldsymbol{K}=\mathcal{C}^{T} \hat{\boldsymbol{K}} \mathcal{C}$ for some reducedorder matrices $\hat{\Lambda}, \hat{\boldsymbol{K}}$, then the co-state equations (24) will not depend on the distributed part of the state $\boldsymbol{\xi}(t)$, and therefore the resulting optimal control can be implemented based on boundary states only. When the cost functional is in the complete form (18) and includes distributed states, a distributed observer may be considered [22], [23] for the estimation of such states.

The implementation of the controller (25) requires in general the discretization of the adjoint equation (24). However, the dual structure of the operator $\mathcal{A}$ and its adjoint implies that any discretization of the state equation automatically provides a natural discretization of the co-state equation, either by modal decomposition or by finite-elements approximation. The 
optimal control is then implementable by solving the two-point boundary value problem given by the interconnection of state and co-state equations, for example using a shooting method provided the initial system state is fully known [24, Section 6].

\section{Simulation Results}

For simulation purposes, a flexible robotic arm (1-2) has been considered with the following physical parameters ${ }^{2}$ :

$$
\begin{gathered}
L=0.7[\mathrm{~m}], \rho=2.975[\mathrm{Kg} / \mathrm{m}], \\
E I=2.4507\left[\mathrm{~N} \cdot \mathrm{m}^{2}\right], J_{h}=0.00195\left[\mathrm{Kg} \cdot \mathrm{m}^{2}\right], \\
M_{p}=0.1117[\mathrm{Kg}], \quad J_{p}=0.0005\left[\mathrm{Kg} \cdot \mathrm{m}^{2}\right]
\end{gathered}
$$

The motion control task is a rest-to-rest maneuver (with zero boundary velocities) that should transfer, within a fixed time interval $T$, the flexible arm from the initial configuration $z(0, x) \equiv 0$ to a desired one $z(T, x)=z_{d}(x)=x \pi / 4$ (a new equilibrium), corresponding to a joint angle $\theta_{d}=\pi / 4$ (set-point) with zero deflection $\delta(T, x) \equiv 0, \forall x \in[0, L]$. Transferring in finite time the flexible arm from the joint position $\theta(0)=0$ to a final joint position $\theta(T)=\theta_{d}$ without inducing any link deflection during motion is physically unfeasible. Therefore, we expect a trade-off between joint error, overall link deflection and total control effort. The final time has been set to $T=8 \mathrm{~s}$, but this does not prevent the optimal trajectory from converging earlier to its steady-state. The weighting matrices in the cost functional (18) have been chosen as follows:

$$
\boldsymbol{\Lambda}=\operatorname{diag}\{0,0,50,10,0,0,1,1\}, \boldsymbol{\Gamma}=\operatorname{diag}\{10,1\}, \boldsymbol{K}=\mathbf{0}
$$

The weights in the first matrix reflect the priority in controlling the position of the payload at the tip of the flexible arm vs. the position of the joint at the link base. In fact, the task priorities are scheduled as follows:

1) $z(t, L) \rightarrow z_{d}(L)=L \theta_{d}$

2) $z_{x}(t, 0)=\theta(t) \rightarrow \theta_{d}$

3) $\dot{z}_{x}(t, 0)=\dot{\theta}(t) \rightarrow 0, \quad \dot{z}(t, L) \rightarrow 0$

Moreover, the non-uniform weights in the input matrix $\Gamma$ correspond to a scenario where the tip control force $F$ is cheaper than the joint torque $\tau$ generated by the motor. We denote by $\boldsymbol{v}_{\mathrm{opt}, L Q}(t)$ the optimal control law given by (25).

In the present case study, we have considered for comparison another control law, denoted for simplicity $\boldsymbol{v}_{P D / F}(t)$, which consists of a standard PD controller for the joint torque, i.e., $\tau_{P D}(t)=k_{p}\left(\theta_{d}-z_{x}(t, 0)\right)-k_{d} \dot{z}_{x}(t, 0)$, with gains $k_{p}=5$ and $k_{d}=3$, together with a stabilizing linear state-feedback for the tip force $F(t)=-k_{F} \dot{z}(t, L)$. Such controller is a modification of the PD scheme proposed in [1, Section 6], and is tuned in order to have a fast response on the prioritized variables $z(t, L)$ and $z_{x}(t, 0)$. To enrich the comparison, we considered also the simple PD version of the controller $\boldsymbol{v}_{P D}(t)$, i.e. with $k_{F}=0$. The PDE system has been simulated using a finite element scheme, i.e., considering a uniform decomposition of $[0, L]$ in $n=10$ elements.

Figures 2-4 compare the performance over time of the two controllers. The LQ control guarantees optimality, and Figure 2 shows that a faster transient is obtained both on joint

\footnotetext{
${ }^{2}$ The considered parameters correspond to the forearm's model of the Flexarm robot [8].
}

and tip positioning, while steady-state accuracy is comparable with the one obtained with the PD/state feedback control and better than the one obtained with the pure PD control that yields undesired oscillations. Furthermore, Figure 3 shows that the arm deformation at the tip is much smaller with the LQ optimal control, in particular in terms of the maximum peak which is less than halved w.r.t. PD/state feedback control, the latter being also characterized by a longer persistency of the deformation. Note that the initial time behaviour of the tip deformation is opposite to and lags behind rigid motion, thus revealing the non-minimum phase nature of this system output. We also found that the initial deformation becomes larger in the reverse scenario of optimal control, when tip force becomes more expensive than joint torque. Figure 4 reports the control inputs. The tip force input $F$ for the LQ optimal control has an initial peak (out of scale) and then a crossover with the joint torque occurs at $t \approx 0.5 \mathrm{~s}$. In particular, the two inputs have opposite signs for a while and display a rather opposite action in order to keep the deformation small. The tip force input $F$ for the $\mathrm{PD} /$ state feedback control is instead characterized by persistent oscillations after the convergence of the states. Evaluating the cost of the three controllers yields

$$
\bar{W}\left(\boldsymbol{v}_{\mathrm{opt}, L Q}\right) \approx 1.8, \quad \bar{W}\left(\boldsymbol{v}_{P D / F}\right) \approx 3.9, \quad \bar{W}\left(\boldsymbol{v}_{P D}\right) \approx 2.9
$$

showing that the LQ optimal control is indeed much cheaper than the PD/state feedback control law. The time history of the cost function along system trajectories in the three considered cases is reported in Figure 5 for a better comparison. We also found that optimal costs are monotonically decreasing for higher flexural rigidity and increasing for larger payload inertia. Figure 6 shows timed snapshots of the flexible arm motion under the action of the LQ optimal controller. After an initial transient, the flexible arm does not show any further appreciable deformation.

\section{Conclusions}

A class of linear-quadratic optimal control problems for an infinite-dimensional model of a single-link flexible robotic arm has been addressed. The cost function evaluates both the distributed and the boundary parts of the system state, together with the effort of two boundary inputs given by the joint torque at the link base and a force at the tip. The optimal controller has been obtained in closed form in terms of the co-state of the system, which is the solution of a suitable adjoint equation. In a case study of a rest-to-rest maneuver, the optimal control law compares favourably with a conventional $\mathrm{PD} /$ state feedback control law.

Future research will be devoted to the extension of the optimal control setup to multi-link flexible robots and oriented towards experimental validation.

\section{REFERENCES}

[1] J. Liu and W. He, Distributed Parameter Modeling and Boundary Control of Flexible Manipulators. Springer, 2018.

[2] A. A. Paranjape, J. Guan, S. Chung, and M. Krstic, "PDE boundary control for flexible articulated wings on a robotic aircraft," IEEE Trans. on Robotics, vol. 29, no. 3, pp. 625-640, 2013.

[3] J.-M. Coron, R. Vazquez, M. Krstic, and G. Bastin, "Local exponential $H^{2}$-stabilization of a $2 \times 2$ quasilinear hyperbolic system using backstepping," SIAM J. on Control and Optimization, vol. 51, no. 3, pp. 2005-2035, 2013 


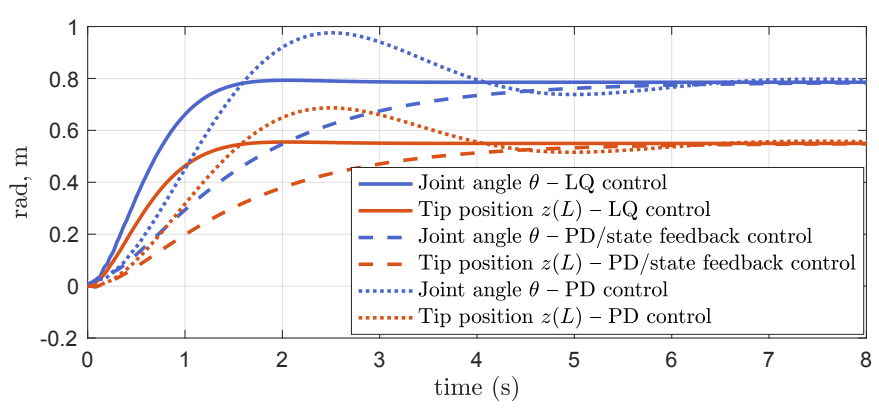

Figure 2. Comparison of LQ optimal control, joint PD and PD/state feedback regulator. Evolution of joint angle $\theta(t)$ and tip position $z(t, L)$.

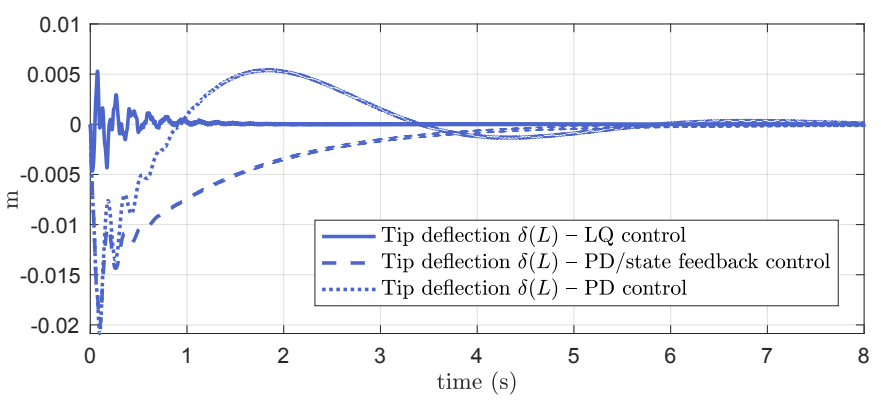

Figure 3. Flexible arm deformation at the tip $\delta(t, L)$ with LQ optimal control, joint PD and PD/state feedback regulator.

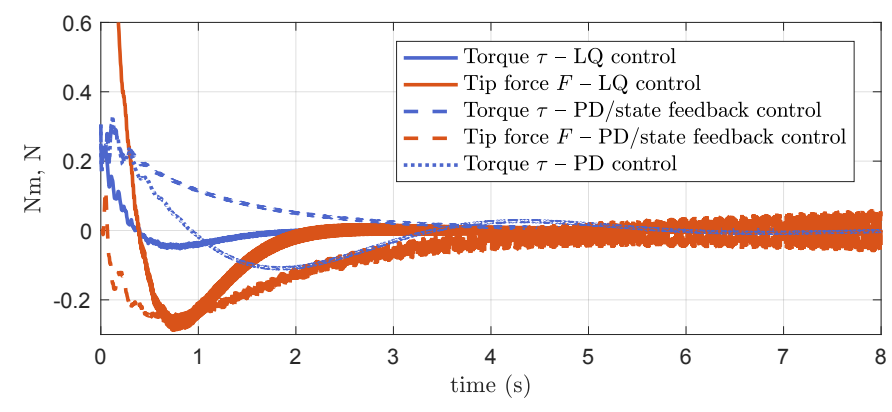

Figure 4. Joint torque $\tau(t)$ and tip force $F(t)$ control inputs with LQ optimal control, joint PD and PD/state feedback regulator.

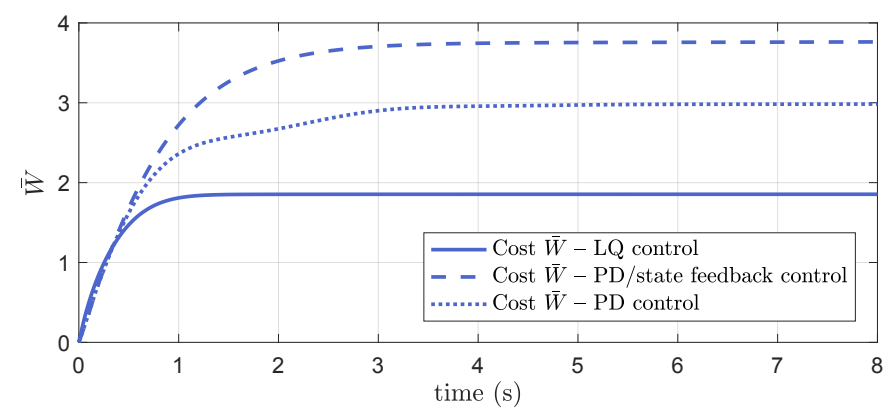

Figure 5. Time history of the cost function for the different control strategies.

[4] A. Hasan, O. M. Aamo, and M. Krstic, "Boundary observer design for hyperbolic PDE-ODE cascade systems," Automatica, vol. 68, pp. 75-86, 2016.

[5] F. Castillo, E. Witrant, C. Prieur, and L. Dugard, "Boundary observers for linear and quasi-linear hyperbolic systems with application to flow control," Automatica, vol. 49, no. 11, pp. 3180-3188, 2013.

[6] M. Barreau, A. Seuret, F. Gouaisbaut, and L. Baudouin, "Lyapunov stability analysis of a string equation coupled with an ordinary differential system," IEEE Trans. on Automatic Control, vol. 63, no. 11, pp. 3850-

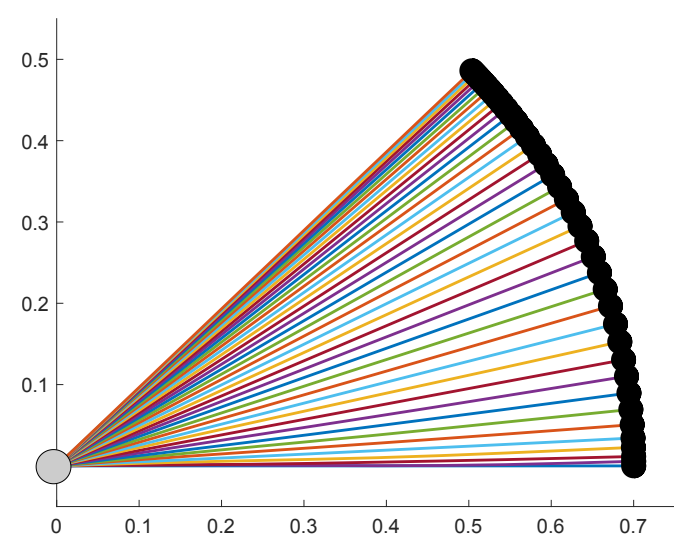

Figure 6. Snapshots of the one-link flexible arm motion in a rest-to-rest maneuver under LQ optimal control (1.5 s of motion sampled every $35 \mathrm{~ms}$ ).

3857, 2018.

[7] F. Ferrante and A. Cristofaro, "Boundary observer design for coupled ODEs-hyperbolic PDEs systems," in Proc. 18th European Control Conf., 2019, pp. 2418-2423.

[8] F. Bellezza, L. Lanari, and G. Ulivi, "Exact modeling of the flexible slewing link," in Proc. IEEE Int. Conf. on Robotics and Automation, 1990, pp. 734-739.

[9] H. Kanoh, "Distributed parameter models of flexible robot arms," Advanced Robotics, vol. 5, no. 1, pp. 87-99, 1990.

[10] J.-H. Park and H. Asada, "Design and control of minimum-phase flexible arms with torque transmission mechanisms," in Proc. IEEE Int. Conf. on Robotics and Automation, 1990, pp. 1790-1795.

[11] A. De Luca and L. Lanari, "Achieving minimum phase behavior in a one-link flexible arm." in Proc. Int. Symposium on Intelligent Robots, 1991, pp. 224-235.

[12] Ö. Morgül, "Orientation and stabilization of a flexible beam attached to a rigid body: planar motion," IEEE Trans. on Automatic Control, vol. 36, no. 8, pp. 953-962, 1991.

[13] A. De Santis and L. Lanari, "Stabilization and control of a flexible structure continuum model," in Proc. IEEE Conf. on Decision and Control, 1993, pp. 3210-3215.

[14] A. De Luca, L. Lanari, and G. Ulivi, "End-effector trajectory tracking in flexible arms: Comparison of approaches based on regulation theory," in Advanced Robot Control, C. Canudas de Wit, Ed. Springer, 1991, pp. 190-206.

[15] Z.-H. Luo, "Direct strain feedback control of flexible robot arms: new theoretical and experimental results," IEEE Trans. on Automatic Control, vol. 38, no. 11, pp. 1610-1622, 1993.

[16] M. Moallem, R. V. Patel, and K. Khorasani, "An inverse dynamics control strategy for tip position tracking of flexible multi-link manipulators," J. of Robotic Systems, vol. 14, no. 9, pp. 649-658, 1997.

[17] W. He, X. He, M. Zou, and H. Li, "PDE model-based boundary control design for a flexible robotic manipulator with input backlash," IEEE Trans. on Control Systems Technology, vol. 27, no. 2, pp. 790-797, 2018.

[18] R. F. Curtain and H. Zwart, An Introduction to Infinite-Dimensional Linear Systems Theory. Springer Verlag, 2012.

[19] F. Tröltzsch, Optimal Control of Partial Differential Equations: Theory, Methods, and Applications. AMS, 2010.

[20] J. Gibson and A. Adamian, "Approximation theory for linear-quadraticgaussian control of flexible structures," SIAM J. on Control and Optimization, vol. 29, no. 1, pp. 1-37, 1991.

[21] J.-P. Raymond, Optimal Control of Partial Differential Equations, Lecture Notes, https://www.math.univ-toulouse.fr/ raymond/bookficus.pdf.

[22] D. Wang and M. Vidyasagar, "Observer-controller stabilization of a class of manipulators with a single flexible link," in Proc. IEEE Int. Conf. on Robotics and Automation, 1991, pp. 516-521.

[23] T. Nguyen and O. Egeland, "Infinite dimensional observer for a flexible robot arm with a tip load," Asian J. of Control, vol. 10, no. 4, pp. 456461, 2008.

[24] D. E. Kirk, Optimal control theory: an introduction. Courier Corporation, 2004. 